\title{
Long-Range Order in the Dislocation Structure of Martensite Crystals
}

\author{
Faina Fedorovna Satdarova \\ Physical Metallurgy and the Physics of Strength Department, National University of Science and Technology, "MISIS”, Moscow, Russia \\ Email address: \\ 2636674@infoline.su

\section{To cite this article:} \\ Faina Fedorovna Satdarova. Long-Range Order in the Dislocation Structure of Martensite Crystals. Advances in Materials. \\ Vol. 9, No. 2, 2020, pp. 28-34. doi: 10.11648/j.am.20200902.12
}

Received: May 16, 2020; Accepted: June 1, 2020; Published: June 17, 2020

\begin{abstract}
Optimal estimation of the diffraction observations over the object reliably detects periodicity in the dislocation structure of martensitic transformation as an exhibition of its wave nature. The period along normal to the slip planes is comparable with the radius of dislocation loops in crystals. The measured degree of one-dimensional long-range order in the arrangement of the loops is close to the upper limit equal to unity. Subject to the theory of metals, the observed structure could be generated by quantum lattice vibrations, which actuate a jump-like phase transition. A simple explanation exists: after a sharp fall in temperature, the excess energy of conduction electrons causes the crystal to expand instantly with the transformation of translational symmetry. Internal shifts of the crystal lattice caused by electron-phonon interactions concurrently trigger the wave process of formation of thin martensitic plates in the surrounding matrix, which are observed in metallography. Based on an in-depth analysis of the dislocation structure of martensite crystals, a physically founded concept is advanced in which the martensitic transformation is a macroscopic quantum phenomenon connected with the symmetry properties of a crystal system in metals.
\end{abstract}

Keywords: System of Dislocation Loops, Ordering by Parallel Slip Planes, Relaxation Vibrations of Crystal Lattice, Quantum Nature of Martensitic Transformation

\section{Introduction}

The diffraction theory of dislocation systems in the crystals is applicable to the entire space of structures: disorder (chaotic network), short-range order (random clusters) and long-range order (regular placement) [1].

The main propositions of the theory that is basis of the method for studying the dislocation structure of highly distorted crystals are specified in [2].

Analysis of the dislocations system arising in crystals upon martensitic transformation reveals one's nature.

A high concentration of fine dislocation loops indicates that phase transformation the microscopic shifts carry out, together transforming the symmetry of crystals [2].

With the discovering of a strong long-range order in the system of dislocation loops, it becomes cleared that microscopic shifts are generated by relaxation vibrations of the crystal lattice at the moment of equilibrium disturbance.

The observability of the long-range order in the dislocation structure of crystals is substantially restricted. Information is available on the one-dimensional long-range order in the system of small dislocation loops, which is existed in the lower harmonics of diffraction line with large reflection indices $\{H K L\}$.

Measuring the order parameters in the dislocation structure of crystals by method created is presented using the example of carbon steel tetragonal martensite.

\section{Identification of the Ordered System of Dislocations in Crystals}

\subsection{Diffraction Mapping of the One-Dimensional Order by Arrangement of Dislocation Loops}

The equation of diffraction theory for deformed crystals includes averaging over a large statistical ensemble of random dislocation systems with all possible values of the parameters describing the system under given macroscopic parameters, being the density of defects in crystal, the measure of correlation and degree of order in their arrangement [1]. 


\subsubsection{General Form of the Diffraction Equation for the Real Crystal Structure}

Let there be a correlation in the system of dislocations and a long-range order in the arrangement of loops along the normal to the slip planes $n \alpha(\alpha=1, \ldots, p)$. The type of loops $\alpha$ is specified by the slip system, the number of them is $p$. The fraction of all ordered loops with random radii of $\xi$ of their total number in a crystal is a degree of order of $\bar{\eta}$.

The periodic component will appear in the equation of the diffraction line harmonics [1]:

$$
\left\{\begin{array}{c}
A_{k} \cong e^{-\langle T(k / \mathfrak{x})\rangle} \quad(k=1,2, \ldots), \\
T=T_{0}+(1-\bar{\eta}) T_{1}+T_{2}(\bar{\eta}) .
\end{array}\right.
$$

Here, T0 corresponds to a random distribution of defects, $\mathrm{T} 1$ takes into account the correlation, T2 represents the longrange order. Angular brackets denote averaging over a statistical ensemble of dislocation systems. An observed fraction of theoretical interval in the diffraction space is æ.

When calculating the periodic component T2 $(\bar{\eta})$, the summation is carried out over the basis vectors of the reciprocal lattice of ordered defects $g=\left(\frac{2 \pi}{\ell}\right) \mathrm{j}$, where $\ell$ is the period of the main translations, $\mathrm{j}$ is the unit vectors in the directions of translation. The terms have a factor $\left(\frac{a}{\ell}\right)^{3}$, which takes into account the number of cells of the reciprocal lattice of ordered defects per unit volume of the reciprocal space of a crystal with a lattice period of $a$.

It is assumed that the distribution of loop sizes of $\xi$ and the distribution of order periods of $\ell$ in the statistical ensemble of dislocation systems are mutually independent and the same for all loops.

\subsubsection{Reciprocal Lattice of Ordered Loops by Normal to Slip Planes}

In the model of ordered placement of loops along parallel slip planes, for each type of loop $\alpha$ there is only one translation vector $\mathrm{j} \| \mathrm{n} \alpha(\alpha=1, \ldots, \mathrm{p})$.

The one-dimensional reciprocal lattice of ordered dislocations in a crystal of finite size $\Xi$ can be represented as a periodic wave packet with a limited spectrum. Peaks with an interval of $\langle 1 / \ell\rangle$ and a width of $\Delta \sim(1 / \Xi)$ will appear in the reciprocal space [3]. Angular brackets denote the expected values of parameters in the statistical ensemble of dislocation systems that are assumed the same for loops of all types $\alpha$.

When averaging periodic component T2 $(\bar{\eta})$, the expected value of the function of random variable $1 / \ell$, which probability of deviating from the center of distribution $\langle 1 / \ell\rangle$ decreases rapidly, is calculated using the expansion to the second central moment [4]:

$$
\left\langle(1 / \ell)^{3}\right\rangle \approx \overline{(1 / \ell}^{3}\left[1+3\left(\sigma_{1 / \ell} / \overline{(1 / \ell)}\right)^{2}\right], \quad \sigma_{1 / \ell} / \overline{(1 / \ell)} \leq 0.1 .
$$

Here, $\sigma_{1 / \ell}^{2}$ is the variance of the unknown distribution law for $1 / \ell$.

It is taken into account that, in the area of the theory's limitations the period of inhomogeneity of crystal distortions is much smaller than its size $\Xi(\xi<\ell<0.1 \Xi)$, and the expected $\langle\ell\rangle$ in the ensemble coincides with the average $\bar{\ell}$ over the crystal volume (according to the ergodic hypothesis [5]).

\subsubsection{Restrictions for Observability of Long-Range Order in the Dislocations System}

A state of order in the dislocation structure of crystals is observable if there is a region in the diffraction space where the parameters included in the model of the object are measurable.

An approximate diffraction equation that allows the measurement of order parameters is obtained under the following assumption in the periodic component T2 $(\bar{\eta})[1]$ :

$$
\frac{2 \pi}{\ell}(\mathrm{j} \mathrm{R}) \ll 1,|\mathrm{R}|=\frac{a(k / æ)}{Q_{H K L}}, Q_{H K L}=\sqrt{H^{2}+K^{2}+L^{2}} .
$$

As a result, the long-range order parameters in the dislocation loops system are, in principle, measurable by the lower harmonics of the diffraction line, i.e., at $k$ close to zero, with a large observing interval in $\mathfrak{x}$ and large reflection indices in $Q_{\{\mathrm{HKL}\}}$.

\subsection{Model of Diffraction Observations Providing Optimal Estimating the Parameters of Studied Object}

Having a limited amount of reliable information in the observational data, it is necessary to select in the parameter vector the main parameters to be estimated, the remaining parameters to be only restricted in the region of allowable values.

\subsubsection{The Equation of the Model of the Best Measurement Accuracy of the Main Parameters}

General equation of the diffraction line harmonics has reformed into a model of observations, which makes it possible to measure both the dislocation density and order parameters in the distribution of loops over the slip planes:

$$
\begin{aligned}
& A_{k} \cong f_{k}(\Theta) \quad\left(k<k_{\text {lim }}\right) \text {, } \\
& f_{k}(\Theta)=\exp ?\left[-t e^{\varphi_{0}}-x_{k} e^{\varphi_{1}}-y_{k} e^{\theta_{1}}\left(\theta_{2} e^{\theta_{3}}\right)\right], \\
& t=-C_{0} Q_{H K L}^{2}, \quad x_{k}=C_{1} \mathfrak{Q}_{H K L}(k / æ), y_{k}=1 / 4 C_{2}(k / ?)^{2} ; \\
& \Theta=\left\{\begin{array}{l}
\varphi \\
\theta
\end{array}\right\}, \quad \theta=\left\{\begin{array}{l}
\theta_{1}=\ln [\bar{c}(\bar{\xi} / a)] \\
\theta_{2}=\bar{\eta} \\
\theta_{3}=\ln \left[\overline{(\xi / \ell)^{3}} W\right]
\end{array}\right\}, \varphi=\left\{\begin{array}{l}
\varphi_{0}=\ln \left[\bar{c}(\bar{\xi} / a)^{2} J_{\xi}(\mu / a) v(1-\bar{\eta})\right] \\
\varphi_{1}=\ln \left[\bar{c}(\bar{\xi} / a)^{2} J_{\bar{\xi}}(1+v(1-\bar{\eta}))\right]
\end{array}\right\},
\end{aligned}
$$




$$
W=J_{\xi}^{6} J_{\ell} ; J_{\xi}=\left[1+\left(\sigma_{\xi} / \bar{\xi}\right)^{2}\right], J_{\ell}=\left[1+3\left(\sigma_{1 / \ell} / \overline{(1 / \ell)}\right)^{2}\right] .
$$

The area of definition of the observation model, within which the approximation of the diffraction theory equation is valid, is limited by the harmonics order of klim.

Numerical values of the crystal-geometry coefficients involved in the equation depend on the type of lattice and the slip set [2]. In particular, for $\langle 111\rangle\{110\}$ bcc C $0=16.278295$, $\mathrm{C} 1=8.769899, \mathrm{C} 2=5.411616$.

In the diffraction observations model (1), the vector of parameters $\Theta$ has two components: $\theta$ - determined parameters of the object; $\varphi$ - other parameters of the dislocations system to modeling an object, performing an auxiliary role in optimization.

Both components $\theta$ and $\varphi$ of the vector $\Theta$ being estimated include the average loops concentration $\bar{c}$ and their average radius $\bar{\xi}$. The vector of parameters of a random system of dislocation loops as well contains the parameters of volumetric and normal correlation $(v, \mu)[1]$ and the coefficients of variation both of loop size $\sigma_{\xi} / \bar{\xi}$ and inverse period of the order $\sigma_{1 / \ell} / \overline{(1 / \ell)}$. All of them fell into the auxiliary component $\varphi$.

\subsubsection{Test the Observational Model Allowability Using Linear Regression Estimates}

For crystals with small dislocation loops, the number of harmonics in the region of all limitations of the diffraction observations model (1) can fall to minimum required; therefore, put $\mathrm{klim}=3$.

Let reduce model (1) to the equation for approximation of observations $\mathrm{Yk}=-\ln$ Ak. Taking into account the stability requirement for sample data fluctuations, we construct the regression equation for the lower harmonics $\{\mathrm{Ak}\}(\mathrm{k}<\mathrm{klim})$, excluding the term quadratic in $\mathrm{k}$ that is insignificant here:

$Y_{k} \cong h_{0} z_{k 0}+h_{1} z_{k 1} \quad\left(\mathrm{z}_{k 0} \equiv t, \mathrm{z}_{k 1} \equiv x_{k}\right)$.

For a sample of data of volume $n$, it is calculated the weighted (with weight $w_{k}=\left(\sigma_{A_{k}}^{2} / A_{k}^{2}\right)^{-1}$, where $\sigma_{A_{k}}^{2}$ is the variance of the measured $\mathrm{Ak}$ ) the sum of the squared deviations from the regression equation of $U$. If by residual deviations the minimal $U^{*}>(2 n-2) /(1-P)$, then the applicability of model (1) is rejected with a confidence of at least $P[6]$.

If the regression equation approximates the data well, the coefficients (h0, h1) are positive and statistically significant then the model of observations is applicable for estimating the parameter vector of $\Theta$.

Estimates of the regression coefficients, being the best in accuracy and stability, give initial approximations for auxiliary component $\varphi$ of the vector $\Theta$.

\subsection{Method of Statistical Estimating the Model of Diffraction Observations}

Different approximations of the theory variously distort the calculated lower harmonics Ak with respect to the true harmonics Âk. It can be assumed that in reality, on a substantially limited interval $(1 \leq \mathrm{k} \leq \mathrm{klim})$, systematic errors of the predicted harmonics of Ak are close to uniform.

\subsubsection{The Objective Function of the Maximum Likelihood of the Data Sample}

Suppose that the squares of variations from true harmonics $\hat{\mathrm{A} k}$, which are added of data errors and model errors, are proportional to the variance of measurements $\sigma_{k}^{2}$ with a roughly constant unknown factor $\zeta$. Let's redefine the problem as estimation by sampling $\{\mathrm{A} r\}(\mathrm{r}=1, \ldots, \mathrm{n})$ with a covariance matrix

$$
\zeta \mathrm{V}_{\mathrm{A}}=\zeta\left[\begin{array}{ccc}
\sigma_{1}^{2} & \cdots & 0 \\
\vdots & \ddots & \vdots \\
0 & \cdots & \sigma_{k_{\mathrm{lim}}}^{2}
\end{array}\right]
$$

When a normal distribution of the sample with a covariance matrix proportional to unknown factor, the maximum likelihood method leads to the following objective function of model optimization [6]:

$$
\left.\begin{array}{c}
L(\boldsymbol{\Theta})=\frac{n m}{2} \log \sum_{r=1}^{n} \sum_{k=1}^{m}\left|\varepsilon_{k, r}(\boldsymbol{\Theta})\right|^{2} / \sigma_{k}^{2}, \\
\varepsilon_{k, r}(\boldsymbol{\Theta})=A_{k, r}-f_{k}(\boldsymbol{\Theta}) .
\end{array}\right\}
$$

Here, $\mathrm{n}$ is the sample size of original data; $\mathrm{m}=\mathrm{klim}$ is dimension of the limited observations vector $\{\mathrm{Ak}\}$.

\subsubsection{The Optimizing Sequence in the Allowable Region of Parameters}

The matrix of the second derivatives of the objective function $-\mathrm{H}$ and the gradient vector $-\mathbf{g}$ are calculated at once with the coefficient $\zeta$, which adjusts the model in the process of approaching the optimum point $\Theta *[6]$ :

$$
\begin{gathered}
\boldsymbol{\Theta}^{i+1}=\boldsymbol{\Theta}^{i}-\mathbf{H}^{-1} \mathbf{g}(i=0,1, \ldots), \\
\mathbf{H}=2 \sum_{r=1}^{n} \sum_{k=1}^{m}\left(\partial f_{k} / \partial \boldsymbol{\Theta}\right)^{\mathrm{T}} w_{k}\left(\partial f_{k} / \partial \boldsymbol{\Theta}\right), \\
\mathbf{g}=-2 \sum_{r=1}^{n} \sum_{k=1}^{m}\left(\partial f_{k} / \partial \boldsymbol{\Theta}\right)^{\mathrm{T}} w_{k} \varepsilon_{k, r}(\boldsymbol{\Theta}), \\
w_{k}(\zeta)=\frac{n m}{2 \sigma_{k}^{2}}\left[\sum_{r=1}^{n} \sum_{k=1}^{m}\left|\varepsilon_{k, r}(\boldsymbol{\Theta})\right|^{2} / \sigma_{k}^{2}\right]^{-1}(1 \leq k \leq m) .
\end{gathered}
$$

The search for optimum is carried out in the region of allowable values of the parameters of the object model that 
are uniquely associated with the vector $\theta$ :

$$
\left\{\begin{array}{l}
10^{8}<\rho_{\mathrm{d}}=\bar{c}(\bar{\xi} / a)\left(2 \pi / a^{2}\right)<10^{12} \mathrm{~cm}^{-2}, \\
0<\bar{\eta}<1, \quad 0.1<\overline{(\xi / \ell)}<1, \\
0<\sigma_{\xi} / \bar{\xi}<0.3, \quad 0<\sigma_{1 / \ell} / \overline{(1 / \ell)}<0.1 .
\end{array}\right.
$$

This includes information from the theory that the diffraction line is detectable when the period of ordered placement of the loops is in the $\bar{\xi}<\bar{\ell}<0.1 \Xi$ interval, under the assumption of a weakly inhomogeneous distortions field in a crystal of size $\Xi[1]$.

To remain within the allowable region, moving from a random starting point $\theta 0$, we introduce a parameter transformation:

$$
\theta_{q}=\frac{1}{2}\left[\underline{\theta_{q}}+\overline{\theta_{q}}\right]+\frac{1}{2}\left[\overline{\theta_{q}}-\underline{\theta_{q}}\right] \sin \omega_{q}(q=1,2,3) .
$$

Operating vector of parameters $\boldsymbol{\omega}$ can vary in infinite limits.

For starting point $\varphi 0$, we choose random variables in intervals of deviations against the regression coefficients: $h_{0} \pm \sigma_{h_{0}}, h_{1} \pm \sigma_{h_{1}}$.

Having a good approximation, it is possible to optimize $\varphi$ without limitations since the objective function (2) will start to grow earlier than the step regulated by the coefficient $0<\gamma^{(i)} \leq 1(\mathrm{i}=0,1, \ldots)$ [6] will reach boundary of the allowable region: $e^{\varphi_{0}}>0, e^{\varphi_{1}}>0$.

\subsubsection{Statistical Decisions on Optimization Results}

When the optimum point $\Theta *$ will reached, it is necessary testing that the model is consistent with the data and that the model parameter estimates are statistically significant.

The criterion for testing residual deviations of the data from the model predictions includes correction on bias:

$$
\eta=(n-(l+1) / m)^{-1} \sum_{r=1}^{n} \sum_{k=1}^{m}\left|\varepsilon_{k, r}\left(\boldsymbol{\Theta}^{*}\right)\right|^{2} / \sigma_{k}^{2} .
$$

Since in $(\mathrm{n} \times \mathrm{m})$ model equations there are $(1+1)$ fitting coefficients ( 1 is the dimension of the vector of estimated parameters $\Theta$ ), the minimum required data sample size is $n>(l+1) / m$.

The model will be rejected as not corresponding to the data with a reliability of at least $\mathrm{P}$ if $\eta>m /(1-P)$ [6].

At a good agreement of the model with observational data, the matrix $\mathrm{H}-1(\Theta *)$ approaches the covariance matrix of the parameters $\mathrm{V} \Theta$ [6] suitable for testing the statistical significance and uncorrelation of estimates in the vector $\Theta *$. Acceptable estimates are considered as measured values of the object model parameters.

Running computational experiments with a random choice of an allowable starting point $\Theta 0$, we accumulate a sufficient sample of measurements to produce the confidence intervals for the dislocation density $\rho_{\mathrm{d}}=e^{\theta_{1}} \cdot \frac{2 \pi}{a^{2}}$ and a degree of order $\bar{\eta}=\theta_{2}$, as well as the lower confidence limit for the period of order, since $\overline{(\xi / \ell)}<e^{\theta_{3} / 3}$.

Approximate confidence intervals, self-corrected over a sample of parameter measured values, are constructed using the method described in [1].

\subsection{Verification of Accuracy of the Parameter Estimates Using Simulation Experiments}

The diffraction line $\{112\}$, best on observability of the long-range order in bcc crystals, was chosen, that interval is $æ=0.2$ from the theoretical period. The main parameters of the model crystal are given in Table 1.

The measured harmonic values of the diffraction line of Ak are simulated by independent normally distributed random variables with theoretically calculated expectation Âk and with the equal standard variation $\sigma=0.01$. When generating data for model crystals using exact diffraction equations [1], the averaging over an ensemble of dislocation systems is carried out using the Monte Carlo method. For simulation tests, a sample of the smallest possible volume in a real experiment of $\mathrm{n}=4$ was generated.

Table 1 represents the results of measuring the parameters of model crystals by lower harmonics of the diffraction line $(\mathrm{klim}=3)$. There are given the average confidence intervals for parameters being determined, which constructed along 60 independent random samples of volume 60, extracted from a total sample of 900 measured

\begin{tabular}{|c|c|c|c|}
\hline \multirow{2}{*}{ Model crystal } & \multirow{2}{*}{ Specified order parameters } & \multicolumn{2}{|c|}{ Approximate $90 \%$ confidence intervals of estimates } \\
\hline & & Dislocation density, $\rho d\left[\mathrm{~cm}-{ }^{2}\right]$ & Degree of order, $\bar{\eta}$ \\
\hline $\operatorname{bcc}(\mathrm{Fe})$ & $\bar{\eta}=0.2, \quad \bar{\ell}=3 \bar{\xi}$ & {$[1.5 ; 2.2] \cdot 1011$} & {$[0.22 ; 0.36]$} \\
\hline$\langle 111\rangle\{112\}$ & $\bar{\eta}=0.5, \bar{\ell}=2 \bar{\xi}$ & {$[1.7 ; 2.6] \cdot 1011$} & {$[0.45 ; 0.61]$} \\
\hline$\rho \mathrm{d}=1011 \mathrm{~cm}-^{2}$ & $\bar{\eta}=0.8, \bar{\ell}=\bar{\xi}$ & {$[2.5 ; 4.1] \cdot 1011$} & {$[0.71 ; 0.82]$} \\
\hline $\bar{\xi} / \mathrm{a}=25$ & - “- & {$[1.0 ; 1.4] \cdot 1011+$} & - \\
\hline
\end{tabular}
values of the vector $\Theta *$.

Table 1. Parameter estimates for the system of dislocation loops with the long-range order by simulation measurements data.

+ Estimation of dislocation density in neglect of long-range order by the higher harmonics of the diffraction line $\{110\}(æ=0.1)$.

The order states in the dislocation structures of model crystals are distinguishable, despite bias of estimates. Just 
because of the estimates bias, the lower confidence limits to periods of the order overlap when $\bar{\ell}>2 \bar{\xi}$.

\subsection{Notes on the Identifiability of a System of Dislocations with Ordering}

Method of determining the long-range order better fit for real crystals. In actuality, the deviations of the predictions according to model (1) with respect to the true lower harmonics of the diffraction line are made more uniform.

For small $k / æ$, the approximation error of the exact diffraction equation constructed by the model of object is smaller. But there the errors of the object model itself, with an approximate description of the displacement field created by dislocation loops in the crystal, have a greater action.

With split dislocations, the errors of the object model become unacceptable for calculating the lower harmonics of the diffraction line of crystals.

In a practical example of martensite of high-carbon alloyed steel, the effect of the split of twinning dislocations is so significant that a regression analysis of the data rejects the observations model (1). The regression curve shows that with the occurrence of micro-twins, the harmonics of the components of $\{112\}$ multiplet for $\mathrm{k}<3(\mathfrak{x}=0.22)$ decrease steeply, then the harmonics attenuation slows down, as in model crystals with a long period of order $(\bar{\ell} / \bar{\xi}>3)$.

Parametric identification of long-range order in the dislocation structure of martensitic crystals with micro-twins is not realizable.

When the method of identifying a system of dislocations by the higher harmonics of the diffraction line described in [1], the effects of long-range order, if they are significant, are added to the systematic errors of the observations model, and identification is adapted to them, which is confirmed by the estimate of the density of dislocations in Table 1.

\section{Dislocations System Determined in Tetragonal Crystals of Steel Martensite}

\subsection{Object Parameters Measurable in Different Areas of Diffraction Space}

When a tetragonal lattice of crystals, in the diffraction observations model (1) the independent variables are transformed:

$$
\begin{aligned}
& \begin{cases}t=-\frac{c}{a}\left[C_{0}+\Delta C_{0}\right] Q_{H K L}^{2}, & \Delta C_{0}=12.4 r+7.75 r^{2}, \\
x_{k}=\frac{c}{a}\left[C_{1}+\Delta C_{1}\right] Q_{H K L}(k / æ), & \Delta C_{1}=1.26 r \\
y_{k}=1 / 4 \frac{c}{a}\left[C_{2}+\Delta C_{2}\right](k / æ)^{2}, & \Delta C_{2}=5.20 r ;\end{cases} \\
& Q_{H K L}=\sqrt{H^{2}+K^{2}+\left(\frac{a}{c}\right)^{2} L^{2}}, \quad ?=\left(\frac{a}{a_{0}}\right) æ\left(a_{0}\right), \\
& 0<r=\left(\frac{c}{a}\right)-1<0.09 \text {. }
\end{aligned}
$$

Here, $\left(\frac{c}{a}\right)$ is the degree of tetragonality, and $a_{0}$ is the period of cubic lattice of crystals. Numerical values of the crystal-geometry coefficients for bcc crystals are given above. (With one more slip kind $\langle 111\rangle\{112\} \mathrm{C} 1=9.493694, \Delta \mathrm{C} 1=$ $2.12 r$.)

At existing tetragonality, the measured dislocation density is $\rho_{\mathrm{d}}=e^{\theta_{1}} \cdot \frac{2 \pi}{a^{2}}\left(\frac{a}{c}\right)$, where $\theta 1$ is component of the parameters vector $\Theta$ in the model of observations (1).

In [2], the method of optimal estimation of harmonics of an approximate shape of lines forming a diffraction multiplet is presented.

Higher harmonics of the identified components of the $\{110\}$ multiplet make it possible to measure the dislocation density $\bar{\rho}_{\mathrm{d}}$ and determine the upper confidence limit of the average loops radius $\bar{\xi}[2]$.

By lower harmonics of the components of the multiplet $\{112\}$, together with the dislocation density, the degree of order $\bar{\eta}$ is estimated and the lower confidence limit of the order period $\bar{\ell} / \bar{\xi}$ is determined.

\subsection{The Received Information on the Parameters of the Object under Study}

The optimal diffraction experiment for structural analysis is described in [1]. X-ray parameters of the test steel sample with a carbon concentration of $\leq 1.0 \mathrm{wt} . \%$ are the same as in [2].

Figure 1 shows dispersion of measured harmonics of the line profile in the diffraction multiplet $\{112\}$ (sample size is $\mathrm{n}=20)$. The harmonics $\{\mathrm{Ak}\}(k \leq 6)$ are statistically significant with a confidence of $99.9 \%$ by the estimate of true variance $\sigma_{A_{k}}^{2}$ [1]. The regression curve in Figure 1 notices the interval of observability of the long-range order in the system of dislocations.

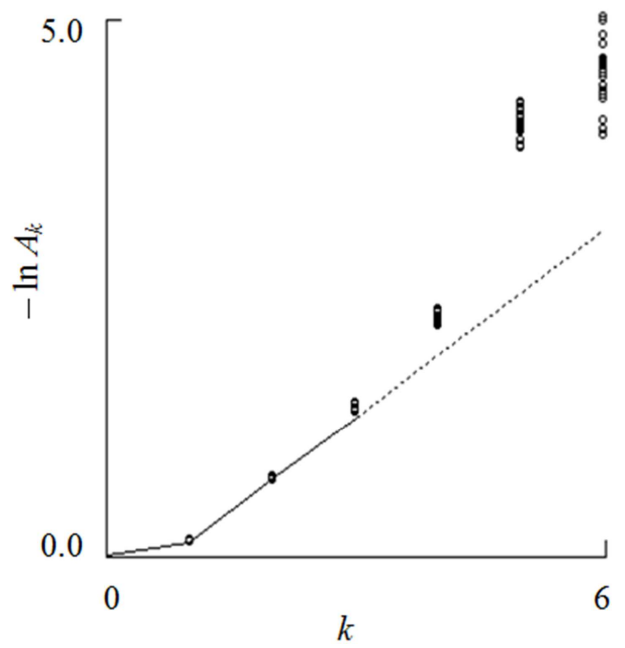

Figure 1. The dispersion of harmonics of approximate shape of lines in the model of martensitic multiplet $\{112\}$ of carbon steel specimen $(\alpha=0.18)$.

Based on reliable data from the analysis of $\{112\}$ diffraction multiplet of carbon steel specimen, well-defined 
parameters of long-range order in the dislocation structure of the martensitic transformation were obtained.

To measure parameters, computational experiments are performed on the model (1); they represent oneself a search for the optimum of the objective function (2) from random starting points uniformly distributed in the allowable region of the object's parameter space.

Analysis of the observational data and estimation of the object parameters were carried out using the updated diffraction study automation system, which is an addition to the monograph [1].

Figure 2 shows the distribution of the measured values of the degree of order in the arrangement of the dislocation loops over parallel slip planes. The size of accumulated data sample is $9 \times 103$.

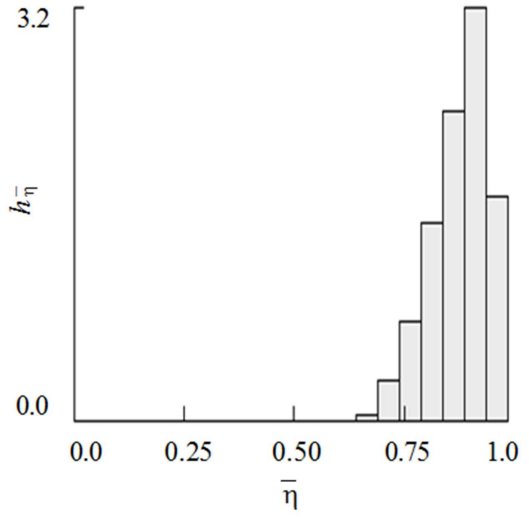

Figure 2. Sampling distribution of measured values of the degree of order in the dislocation structure of martensite crystals of carbon steel.

Table 2. Long-range order in the dislocation structure of the martensitic transformation of carbon steel.

\begin{tabular}{lll}
\hline Measured parameter & Approximate 90\% confidence interval of estimates & The most plausible estimates by histogram maxima \\
\hline Dislocation density, $\bar{\rho} \mathrm{d}\left[\mathrm{cm}-^{2}\right]$ & {$[0.7 ; 0.8] \times 1012[0.6 ; 0.7] \times 1012+$} & $(0.6-1.0) \times 1012$ \\
Degree of order, $\bar{\eta}$ & {$[0.88 ; 0.91][0.85 ; 0.88]+$} & $(0.90-0.95)$ \\
\hline
\end{tabular}

+ Estimates by the lower harmonics of the components within multiplet $\{110\}$.

Table 2 presents the average confidence intervals for parameters being measured, which constructed on 900 random samples of volume 60 extracted from the total sample of measurements.

The estimate of the dislocation density corresponds to that expected from analysis of the higher harmonics of the diffraction multiplet $\{110\}[2]$.

The lower harmonics of the components of $\{110\}$ multiplet under large measuring interval $(æ=0.23)$ on a double-quenched sample with fallen the fraction of residual austenite to $<4 \%$, gave close interval estimates of the order parameters, and the most plausible estimates are the same as those from the data of analysis of multiplet $\{112\}$ (Table 2).

With a reliability of at least $90 \%$, the lower confidence limit of the order period in the system of dislocation loops is $\bar{\ell} / \bar{\xi}>\simeq 1$. That estimate is confirmed by the lower harmonics of the components of multiplet $\{110\}$.

The appearance of a long-range order along normal to the slip planes containing clusters of dislocation loops, in the continuum approximation, is clarified by a wave of vibrations of an elastic medium. According to the equation of movement of an elastic medium in a plane perpendicular to the direction of extension of an elastic wave, displacements are purely shear, just like in slip planes in a crystal [7].

\section{Conclusion}

In crystals of martensite, the periodic dislocation structure revealed itself, giving rise to an image of a hardened wave of lattice vibrations.

It is known the process of emission of long-wavelength phonons (quanta of lattice vibrations) by "hot" electrons in low-temperature relaxation of the system. [8].

If the observed period of order $\ell$ is appropriate to the phonon wavelength, then the dislocation loops of comparable radius $\xi \sim \ell$ extinguish phonons, which originated them [9].

There arises an idea of a quantum phase transition associated with the transformation of the symmetry of crystals. And it can be verified experimentally.

From sudden thermal disturbance in electron system of a crystal, quanta transitions occur inter energy levels. The phonons excited by them move the lattice to stable state and disappear. In equilibrium, surfaces of equal electron energy, constructed in the reciprocal space of a crystal, should have complete lattice symmetry [10].

(When interstitial carbon atoms are in the crystal lattice, there is a high probability of local electron-phonon interactions as well [11].)

An external magnetic field rearranges the resolved under the symmetry requirements energy states of electrons [12]; therefore, it should affect the martensitic transformation in all metals.

The theory of quantum martensitic transformation, to which analysis of arising in crystals the system of dislocations has led, in principle, is allowed the experimental verification by known method [13].

\section{Acknowledgements}

The production of test samples and the diffractometric experiments optimal on reliability of the data obtained were performed by a well-qualified professional Dmitry Aleksandrovich Kozlov.

\section{References}

[1] Faina F. Satdarova, Diffraction Analysis of Deformed Metals: Theory, Methods, Programs, Academus Publishing, Inc., California: Campbell, 2020, in press. 
[2] Satdarova F. F. (2016), Dislocation structure of martensitic transformation in carbon steel. Phys. Met. Metallography 117, 355-363.

[3] G. A. Korn and T. M. Korn, Mathematical Handbook for Scientists and Engineers: Definitions, Theorems, and Formulas for Reference and Review. McGraw-Hill, New York, 1968; Nauka, Moscow, 1974.

[4] L. Yanossy, Theory and Practice of the Evaluation of Measurements, Oxford Univ., 1965; Mir, Moscow, 1968.

[5] L. D. Landau and E. M. Lifshitz, Course of Theoretical Physics, Vol. 5: Statistical Physics, 3rd ed., Nauka, Moscow, 1976; Pergamon, Oxford, 1980.

[6] Y. Bard, Nonlinear Parameter Estimation, Academic Press, New York, 1974; Statistika, Moscow, 1979.

[7] L. D. Landau and E. M. Lifshitz, Course of Theoretical Physics, Vol. 7: Theory of Elasticity, 3rd ed., Nauka, Moscow, 1965; Pergamon, Oxford, 1980.
[8] L. D. Landau and E. M. Lifshitz, Course of Theoretical Physics, Vol. 10: Physical Kinetics, Nauka, Moscow, 1979; Pergamon, Oxford, 1981.

[9] C. Teodosiu, Elastic Models of Crystal Defects, SpringerVerlag, Berlin, Heidelberg, New York, 1982; Mir, Moscow, 1985.

[10] J. P. Elliott and P. G. Dawber, Symmetry in Physics, Vol. 1 and 2, Macmillan Press, London, 1979; Mir, Moscow, 1983.

[11] Reich K. V., Eidelman E. D. (2011), Electron-phonon interaction in a local region. Physics of the Solid State 53, 1704-1706.

[12] J. M. Ziman, Principles of the Theory of Solids, Cambridge Univ., 1964; Mir, Moscow, 1966.

[13] Schastlivtsev V. M., Kaletina Yu. V, Fokina E. A. and Mirzaev D. A. (2016), Nature of the effect of magnetic fields on the starting temperature of martensitic transformation in iron alloys. Physics of the Solid State 58, 336-345. 